\title{
Prognostic value of TOP2A in bladder urothelial carcinoma and potential molecular mechanisms
}

Shuxiong Zeng ${ }^{1 \dagger}$, Anwei Liu ${ }^{1 \dagger}$, Lihe Dai ${ }^{1 \dagger}$, Xiaowen $\mathrm{Yu}^{2}$, Zhensheng Zhang ${ }^{1}$, Qiao Xiong ${ }^{1}$, Jun Yang ${ }^{1}$, Fei Liu ${ }^{1}$, Jinshan $\mathrm{Xu}^{1}$, Yongping $\mathrm{Xu} \mathrm{e}^{1}$, Yinghao Sun ${ }^{1 *}$ and Chuanliang $\mathrm{Xu}^{1 *}$

\begin{abstract}
Background: The prognosis of bladder urothelial carcinoma (BLCA) varies greatly among patients, and conventional pathological predictors are generally inadequate and often inaccurate to predict the heterogeneous behavior of BLCA. This study aims to investigate the prognostic value and function of TOP2A in BLCA.

Methods: TOP2A expression level was examined by RNA-sequencing, quantitative real time polymerase chain reaction and immunohistochemistry from 10,40 and 209 BLCA samples, respectively. Public databases were analyzed for validation. Cell proliferation, migration, invasion assays were performed to explore potential functions of TOP2A in BLCA. Flow cytometry was performed for cell cycle and apoptosis analysis. Univariable and multivariable Cox regression models were performed to identify independent risk factors for the prognosis of BLCA.

Results: We found TOP2A was significantly upregulated in BLCA samples, especially for high-grade and advanced stage tumors, compared with matched normal epithelial tissue. Univariable COX regression analysis revealed high TOP2A expression was significantly associated with poorer cancer-specific, progression-free and recurrence-free survival, but not independently of clinical characteristics in the multivariable models.

Knockdown of TOP2A remarkably inhibited the proliferation of BLCA cells and non-cancerous urothelial cells. Furthermore, migration and invasion capacity of BLCA cells were strongly suppressed after TOP2A knockdown. Moreover, flow cytometry suggested TOP2A had anti-apoptotic function, and knockdown of TOP2A could induce resistance to doxorubicin in $J 82$ cells.
\end{abstract}

Conclusions: In our study, TOP2A was overexpressed in BLCA and could serve as a prognostic biomarker for BLCA. Moreover, TOP2A is functionally important for the proliferation, invasion and survival of BLCA cells.

Keywords: Bladder urothelial carcinoma, TOP2A, Prognosis, Biomarker

\section{Background}

Bladder urothelial carcinoma (BLCA) is the most common neoplasm in the urological system in China [1]. Five to $10 \%$ of patients are already at metastatic stage at diagnosis, and around $50 \%$ of patients will develop local or distant disease progression after radical cystectomy

\footnotetext{
* Correspondence: sunyhsmmu@126.com; xuchuanliang@vip.126.com tShuxiong Zeng, Anwei Liu and Lihe Dai contributed equally to this work ${ }^{1}$ Department of Urology, Changhai Hospital, Second Military Medical University, Shanghai, People's Republic of China

Full list of author information is available at the end of the article
}

[2]. Conventional pathological predictors such as tumor stage and grade are generally inadequate and often inaccurate to predict the heterogeneous behavior of BLCA [3]. Identification of recurrent genetic alteration in BLCA is critical for discovering genes that drive BLCA and identifying prognostic biomarkers for stratification of patients with different risks of prognosis [4]. With the advent of next-generation sequencing technology and computational biology, the understanding of BLCA has entered a new era [4]. Gaining insight into the biology of bladder cancer may reveal numerous biomarkers that

(c) The Author(s). 2019 Open Access This article is distributed under the terms of the Creative Commons Attribution 4.0 International License (http://creativecommons.org/licenses/by/4.0/), which permits unrestricted use, distribution, and reproduction in any medium, provided you give appropriate credit to the original author(s) and the source, provide a link to the Creative Commons license, and indicate if changes were made. The Creative Commons Public Domain Dedication waiver (http://creativecommons.org/publicdomain/zero/1.0/) applies to the data made available in this article, unless otherwise stated. 
enhance the sensitivity and specificity of early diagnosis and treatment of BLCA [5].

Topoisomerase-II alpha (TOP2A) is an essential nuclear enzyme which regulates the topological state of DNA during transcription and is involved in the processes of chromosome condensation and chromatid separation [6]. Several studies have reported that higher expression levels of TOP2A are indicative of poor prognosis in a variety of human cancers [6-9]. Moreover, TOP2A is the target for some of the most widely used chemotherapeutic drugs for treatment of human cancers $[10,11]$. Despite the well-known association between TOP2A expression and aggressiveness of different cancers, the underlying role of TOP2A in the BLCA remains unclear.

In the present study, we explored the expression and function of TOP2A in BLCA patient samples and cell lines. We found that TOP2A was overexpressed in BLCA, and higher TOP2A expression was associated with poorer cancer-specific, progression-free and recurrence-free survival. We also revealed that TOP2A regulated proliferation and invasion, and played a role associated with antiapoptosis in BLCA.

\section{Methods}

\section{Clinical samples and cell lines}

Ten pairs of BLCA samples (five muscle-invasive bladder cancer (MIBC) and five non-muscle invasive bladder cancer (NMIBC), eight high-grade and two low-grade) and their matched adjacent normal epithelial tissue were collected for RNA-sequencing (RNA-seq) from patients who were treated with cystectomy from June 2013 to May 2014. Another 40 BLCA samples (21 MIBC and 19 NMIBC, 26 highgrade and 14 low-grade) were collected from transurethral resection of bladder cancer (TURBT) or cystectomy for further validation by real time quantitative polymerase chain reaction (RT-qPCR). A total of 209 formalin-fixed BLCA tumors (130 MIBC and 79 NMIBC, 158 high-grade and 51 low-grade, median age 69 years, 144 males and 65 females, 144 from cystectomy and 65 from TURBT) were collected for immunohistochemistry from July 2008 to December 2015. No patient received chemotherapy or radiotherapy previously in this study. All clinical samples were obtained after written informed consents were provided by the patients, and the study protocol was approved by the Ethics Committee of Changhai Hospital. BLCA cells lines used in this study were purchased from the American Type Culture Collection (Manassas, VA, U.S.) and cells were cultured with the recommended medium and condition. Gemcitabine (Catalog No.S1149) and doxorubicin (Catalog No.S1208) was purchased from Selleck.
Gene expression analysis by RNA-sequencing

RNAs were purified from tumor tissue for RNA-Seq analysis by Beijing Genomics Institute (Shenzhen, China). All samples were examined by pathologists to ensure that tumor sample had tumor density $>80 \%$ and the adjacent normal tissue were without tumor contamination. Experimental procedures of RNA-Seq were described previously $[12,13]$.

\section{Database mining}

Public databases: gene expression omnibus (GEO, GSE31684, $n=93$ ) (http://www.ncbi.nlm.nih.gov/gds/), and two comprehensive studies, i.e. The Cancer Genome Atlas (TCGA, bladder cancer, $n=413$ ) and MSKCC (bladder cancer, $n=97$ ) on BLCA gene expression profile and prognosis in cBioPortal (http:// www.cbioportal.org/) were included for data mining.

\section{RNA extraction and RT-qPCR}

Total RNA was extracted by TRIzol reagent (Invitrogen $\left.^{\mathrm{nt}}\right)$, and the cDNA was synthesized using a PrimeScript RT Reagent kit (Takara, Japan) following the manufacturer's instruction. RT-qPCR was conducted on an Applied Biosystems Step One Plus (Agilent, USA) using SYBR ${ }^{\circ}$ Premix Ex Taq ${ }^{\text {Tw }}$ (Takara, Japan). The relative fold changes were calculated with $2-\Delta \Delta C T$ methods. The primers used are as follows: $5^{\prime}$-CATT GAAGACGCTTCGTTATGG-3' (forward) and 5' - CCA GTTGTGATGGATAAAATTAATCAG-3' (reverse) for TOP2A gene; 5'-ACGGTAGCTGCTAAAAAGGGAA3' (forward) and 5' - GGATGTTTTCCTGCCAGGGT3' (reverse) for TOP2B; 5' - ACCACAGTCCATGCCA TCAC-3' (forward), 5' - TCCACCACCCTGTTGCTG TA-3' (reverse) for GAPDH.

\section{Immunohistochemistry}

Formalin-fixed paraffin-embedded BLCA samples were cut into $5-\mu \mathrm{m}$-thick sections. Primary antibody (1:300) anti-TOP2A (ab52934) and anti-Ki67 (ab15580) was purchased from Abcam ${ }^{\circ}$. Immunohistochemistry staining was conducted according to the instruction of the immunohistochemistry kit (BioGenex, Fremont, CA, U.S.). The immunohistochemical stain of TOP2A was evaluated by pathologists in a blinded fashion and was scored on the percentage of positive tumor cell nucleus (negative, score $0 ;<1 / 3$, score $1 ; 1 / 3-2 / 3$, score $2 ;>2 / 3$, score 3$)$. Scores of $0-1$ were defined as low expression, and 2-3 were defined as high expression.

\section{RNA interference and lentivirus construction}

The small interference RNA (siRNA) against TOP2A (5'GGUCAGAAGAGCAUAUGAUTT-3'; antisense, 5'AUCAUAUGCUCUUCUGACCTT-3' for siRNA-1; sense, 5'-GACCAACCUUCAACUAUCUTT-3'; antisense, 5'- 
AGAUAGUUGAAGGUUGGUCTT-3' for siRNA-2; sense, 5'-GCTGCGGACAACAAACAAATT - 3'; antisense, 5' GCTATCAGCCTGGCCTTTATT-3' for siRNA-3) and non-specific siRNA (sense 5'-UUCUCCGAACGUGUCACGUTT-3', antisense 5'-ACGUGACACGUUCGGAGAATT - 3') were purchased from GenePharma, Shanghai, China. Cells were transfected with siRNA according to the instruction of the manufacturer (Lipofectamine ${ }^{\circ}$ RNAiMAX, Invitrogen). Lentivirus with small hairpin RNA against TOP2A (5'- GATCCGGTCAGAAGAGCATAT GATTTCAAGAGAATCATATGCTCTTCTGACCTTTTT TC-3') and non-specific small hairpin RNA (5' - CTAG CCCGGCCAAGGAAGTGCAATTGCATACTCGAGTAT GCAATTGCACTTCCTTGGTTTTTTGTTAAT-3') were purchased from Hanbio (Shanghai, China). J82 Cells were transfected with lentiviral shRNA at a multiplicity of infection of about 30 in the presence of $5 \mu \mathrm{g} / \mathrm{ml}$ polybrene. Puromycin was added to medium at the concentration of $2.5 \mathrm{mg} / \mathrm{ml}$ for stable cell line selection.

\section{Cell proliferation, wound-healing and Matrigel invasion assays}

Cell proliferation and drug sensitivity assay was conducted per the instruction of Cell Counting Kit-8 (CCK8 ; Dojindo). Cells with a density of $3 \times 10^{3}$ per well were seeded in triplicates in 96-well plates with $200 \mu \mathrm{L}$ medium, and cell viability was determined using CCK- 8 kit by adding $20 \mu \mathrm{L}$ reagent. The cells were incubated for another $2 \mathrm{~h}$ and then the absorbance at $495 \mathrm{~nm}$ was measured with EnSpire Reader. Wound-healing assay was conducted by seeding cells in triplicate in 6-well plates. After the cell density reached about $80 \%$, then $10 \mu \mathrm{L}$ pipette tips were applied to create a cell-free trip area and the floating cells were washed away by PBS buffer. Cells were then cultured with pure medium without $10 \%$ fetal bovine serum (FBS). Photographs of the scratched area were taken at $0 \mathrm{~h}, 12 \mathrm{~h}, 24 \mathrm{~h}$ and $72 \mathrm{~h}$, respectively. Invasion assays were performed with the transwell chamber with Matrigel (Millipore, USA). Cells with a density of $5 \times 10^{4}$ were seeded into the upper chamber and cultured with $500 \mu \mathrm{L}$ pure medium without $10 \% \mathrm{FBS}$, and the lower chamber were added with $1 \mathrm{ml}$ complete medium. After $24 \mathrm{~h}$ or $48 \mathrm{~h}$, cells remaining on the upper membrane were removed with cotton wool, and then placed into $4 \%$ formalin for $20 \mathrm{~min}$. The cells on the membrane were stained with $0.1 \%$ crystal violet for $30 \mathrm{~min}$. Microscope photographs of 10 random filed of the membrane were taken, and cells were counted for further statistical analyses.

\section{Tumor formation in nude mice}

All animal experiments were conducted with the approval of the Scientific Investigation Board of the Second Military Medical University. J82 cells infected with lentivirus against TOP2A and non-specific lentivirus were injected subcutaneously into the lower flank of female athymic BALB/c nude mice. Each group had 6 mice and each mouse was injected with $1 * 10^{6} \mathrm{~J} 82$ cells. Tumor size was measured weekly and tumor volume was calculated using the following formula: Volume $=$ Length $\mathrm{x}$ width ${ }^{2} \mathrm{x}$ $0.5\left(\mathrm{~mm}^{3}\right)$. Mice with tumor diameter $>1.5 \mathrm{~cm}$ or with losses in body weight of $>20 \%$ were promptly euthanized by asphyxiation by $\mathrm{CO} 2$ inhalation from compressed gas source. All animal studies were performed according to the Guidelines of the Second Military Medical University for the Care and Use of Laboratory Animals.

\section{Flow cytometry analysis}

Cells were collected for flow cytometry analysis $72 \mathrm{~h}$ after transfection with TOP2A siRNA or control siRNA. Cells were stained using cell cycle kit (Lianke Biotech Co., Ltd., China) per manufacturer's instructions, and then analyzed on a Beckman Coulter Analyzer (Beckman Coulter, Brea, CA, USA). Cell cycle data were analyzed with Modfit software. Apoptosis was performed using Annexin V fluorescein isothiocyanate and propidium iodide apoptosis kit (Lianke Biotech Co., Ltd., China). Stained cells were analyzed on a MACSQuant Analyzer (Teterow, Germany), and data was then analyzed by FlowJo v7.6.1 software.

\section{Statistics}

At least three independent experiments were performed for each experiment described in this study. Student $t$ test or one-way ANOVA was used to compare continuous parametric data between two groups and multiple groups, respectively. Kaplan-Meier survival curve and log-rank test were applied for survival analysis. Univariable and multivariable Cox proportional hazards models were performed to identify independent risk factors for the prognosis of BLCA. A backward step-down wald selection method was used (the entry with $P<0.05$ and removal criteria with $P<0.10)$. The statistical analysis was performed either by SPSS 19.0 (IBM Inc.) or GraphPad Prism 5 (GraphPad Software, Inc., La Jolla, CA).

\section{Results}

TOP2A was up-regulated in BLCA

We performed RNA-Seq on ten pairs of matched BLCA tumor samples and the adjacent normal tissue obtained by cystectomy. We used a cutoff of $\log 2-$ fold change $>2$ and probability $>0.8$, a method proposed by Tarazona et al. [14], to filter differentially expressed genes between tumor samples and the 
matched normal epithelial tissue (Fig. 1a). We identified TOP2A was up-regulated in nine out of ten tumor samples with a $\log 2$-fold change of 3.02 and probability of 0.86 (Fig. 1b). As shown in Fig. 1b, the average level of TOP2A, represented by reads per gene per kilobase exon per million mapped reads (RPKM), was higher in MIBC than NMIBC. We further validated the expression of TOP2A in an additional 40 tumor samples and the matched normal epithelial tissue using RT-qPCR, the baseline characteristics of the patients were shown in Table 1 . We found that TOP2A was significantly up-regulated in tumor samples (Fig. 1c), especially higher for MIBC (Fig. 1d) and high-grade tumors (Fig. 1e). We further explored the NCBI-GEO datasets to determine the expression levels of TOP2A in BLCA. We found that the TOP2A expression was significantly higher in MIBC compared with NMIBC in GSE31684 dataset, which included 93 patients with high-risk BLCA from Memorial Sloan-Kettering Cancer Center (MSKCC, Fig. 1f).

\section{TOP2A expression correlated with prognosis of BLCA}

We evaluated the expression of TOP2A by immunohistochemical analysis in 209 patients who underwent transurethral resection of BLCA or cystectomy. The baseline characteristics of the patients were shown in Table 1 . The TOP2A showed a totally nuclear expression pattern, and it was almost negative in all normal urothelium that could be studied adjacent to tumor tissue (Fig. 2a). Of the 209 tumor samples, high (staining score, $2.23 \pm 0.42$, Fig. 2b) and low (staining score, $0.95 \pm 0.22$, Fig. 2c) TOP2A expression was found in 99 and 110 patients, respectively. The TOP2A staining score was also significantly higher in high-grade tumor $(1.67 \pm 0.74)$ and MIBC tumor $(1.56 \pm 0.49)$ than low- grade
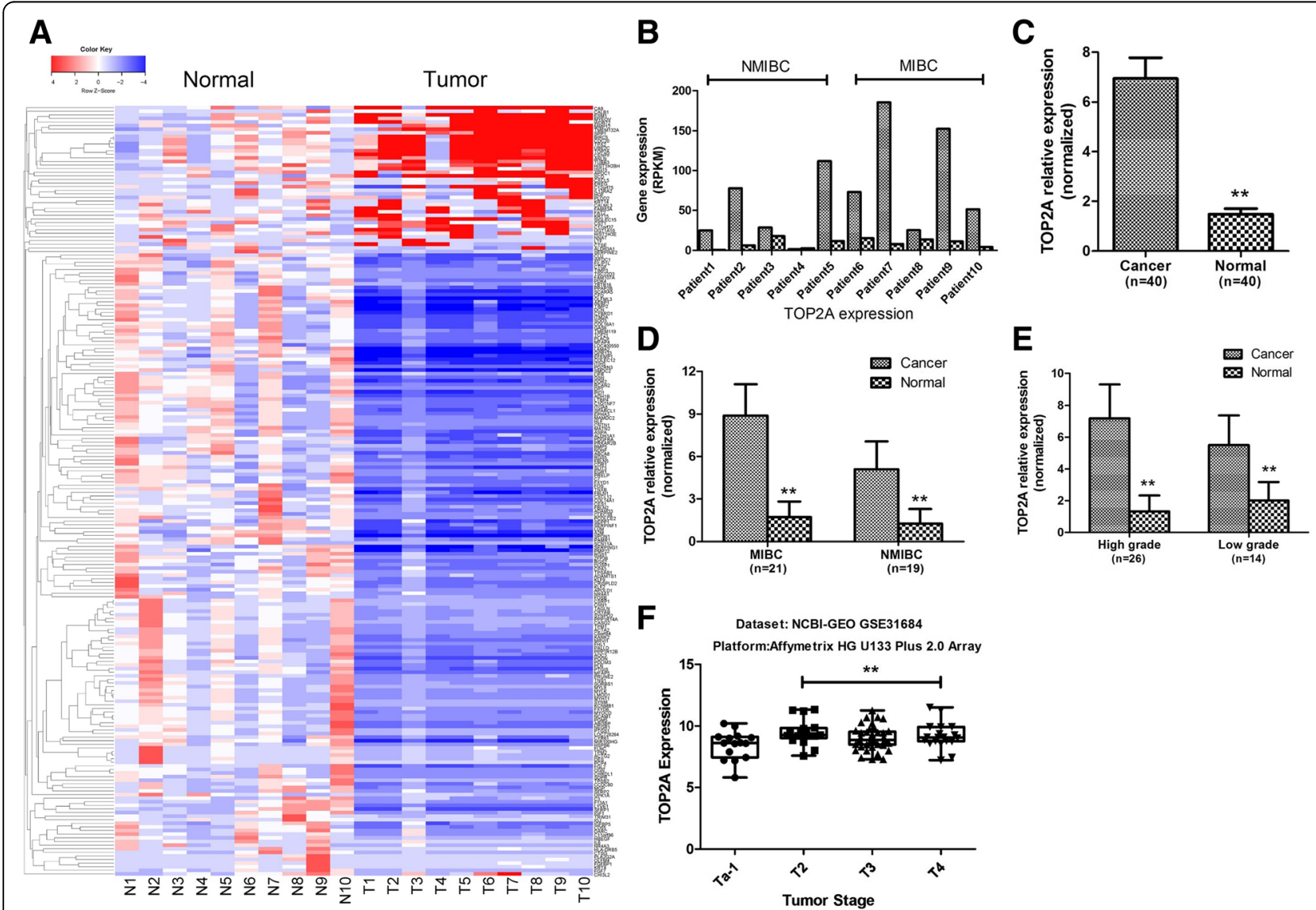

Fig. 1 Increased TOP2A expression in bladder urothelial carcinoma (BLCA) revealed by RNA-sequencing (a) and RT-qPCR (c-e). a Hierarchical clustering heat map of differentially expressed genes between tumor tissue and matched normal epithelial tissue. $\mathbf{b}$ Expression levels of TOP2A in each sample for RNA-sequencing. c TOP2A gene expression was significantly up regulated in tumor tissue compared with matched normal epithelial tissue. $\mathbf{d}$ TOP2A gene expression in different stages of BLCA. e TOP2A gene expression in different grades of BLCA. $\mathbf{f}$ TOP2A gene expression was significantly higher in MIBC compared with NMIBC in NCBI-GEO GSE31684 dataset. MIBC = muscle-invasive bladder cancer; NMIBC = non-muscle invasive bladder cancer; RPKM = reads per gene per kilobase exon per million mapped reads. ${ }^{*}, p<0.01$ 
Table 1 Baseline characteristics of patients for RT-qPCR and IHC validation

\begin{tabular}{lll}
\hline & RT-qPCR cohort $(n=40)$ & IHC cohort $(n=209)$ \\
\hline Age, median(IQR) & $65(56,70)$ & $69(61,75)$ \\
Gender, men/women & $31 / 9$ & $144 / 65$ \\
Surgery type & & \\
TURBT & 12 & 65 \\
Cystectomy & 28 & 144 \\
Pathological stage & & \\
Ta, Cis, T1 & 19 & 79 \\
T2 & 13 & 70 \\
T3 & 6 & 54 \\
T4 & 2 & 6 \\
Grade & 14 & 51 \\
Low & 26 & 158 \\
High & & 198 \\
Lymph node status & 38 & 11 \\
negative & 2 & \\
positive & & \\
\hline
\end{tabular}

RT-qPCR = real time quantitative polymerase chain reaction; $\mathrm{IHC}=$ immunohistochemistry; TURBT = transurethral resection of bladder tumor; $\mathrm{IQR}=$ Interquartile range

tumor $(1.11 \pm 0.43, P<0.001)$ and NMIBC tumor $(1.27 \pm 0.45, P<0.001)$, respectively.

With a median follow-up of 27.9 months (range, 10 to 95 months), patients with high TOP2A expression showed significantly poorer cancer-specific survival (CSS, Fig. 2d, median, 21.3 vs. 41.1 months, $P=0.004$ ), progression-free survival (PFS, Fig. 2e, median, 16.8 vs. 33.6 months, $P=0.008$ ) and recurrence-free survival (RFS, Fig. 2f, 11.6 vs. 37.3 months, $P=0.004$ ) compared with patients with low TOP2A expression. Survival analysis was further investigated on $\operatorname{MIBC}(n=130)$ and NMIBC $(n=79)$ cohorts respectively. In MIBC cohort, patients with elevated TOP2A expression had significantly poorer CSS $(P=0.044)$, PFS $(P=0.049)$ and RFS $(P=0.041)$ compared with patients with low expression (Additional file 1: Figure S1). However, no difference was noticed between TOP2A high expression and low expression groups in NMIBC cohort (Additional file 1: Figure S1). These results suggested that TOP2A had better prognostic value for patients with MIBC. Detailed results of univariable and multivariable Cox regression analysis of predicative variables of CSS were summarized in Table 2. The level of TOP2A was revealed to be associated with CSS in univariable COX regression analysis. However, multivariable COX regression analysis showed tumor stage and lymph node status were independent predictors of CSS. We further revealed that TOP2A expression ran in parallel to Ki67, which was an established proliferation biomarker, with a correlation coefficient of 0.6 (Spearman test with $P<0.01$ ).

\section{The expression of TOP2A mRNA and protein diverged from each other}

We further investigated the association between the levels of TOP2A mRNA and clinical outcome of patients in public databases of TCGA and NCBI-GEO. Patients with TOP2A mRNA expression above 75\% (high expression) and below 25\% (low expression) were included for further analysis [15]. As shown in Fig. 3a, patients with up-regulated TOP2A mRNA showed tendentially shorter recurrence-free survival compared to patients with low expression (27.8 vs. 36.8 months), but it did not reach statistical significance $(P=0.135)$. Regarding the NCBI-GEO (GSE31684, Fig. 3b) and MSKCC (bladder cancer, JCO, 2013, Fig. 2c) datasets, no significant differences were detected between TOP2A mRNA high expression and low expression groups. The expression pattern of TOP2A mRNA and protein was further investigated in 14 BLCA samples by RT-qPCR and western blot, respectively (Fig. 3d and e). We found no association between TOP2A mRNA and protein, with correlation coefficient of -0.40 (Additional file 2: Figure S2, $P=0.15)$.

\section{Functional alteration of BLCA cells after knockdown of TOP2A}

We investigated the level of TOP2A in several bladder cancer cell lines, J82 and 5637 cells had relative higher TOP2A expression (Fig. 4a). To explore the potential function of TOP2A in BLCA, we knocked down TOP2A expression in $J 82$ and 5637 cells using siRNA. The level of TOP2A mRNA and protein were significantly downregulated after siRNA transfection in J82 and 5637 cells, while TOP2B expression was not influenced (Additional file 2: Figure S2). We then analyzed the proliferation rate of J82, 5637 and non-cancerous urothelial cells SVHUC after TOP2A knockdown. As shown in Fig. 4b, cell proliferation rate was significantly inhibited 5 days after TOP2A knockdown in J82 and 5637 cells. Meanwhile, we also observed that cell proliferation rate of SVHUC decreased after TOP2A knockdown (Additional file 2: Figure S2). Additionally, we developed 182 cells with TOP2A stably knocked down using lentivirus-mediated gene transfer method (Additional file 2: Figure S2). We further tested the effect of knockdown of TOP2A expression on growth as subcutaneous xenograft nude mice model. Tumor volume was measured from two to 8 weeks after $\mathrm{J} 82$ cells implantation. The difference of tumor volume between TOP2A knockdown group and control group was statistically significant 6 weeks after tumor cells implantation (Fig. 4c and Additional file 4: Table S1). 

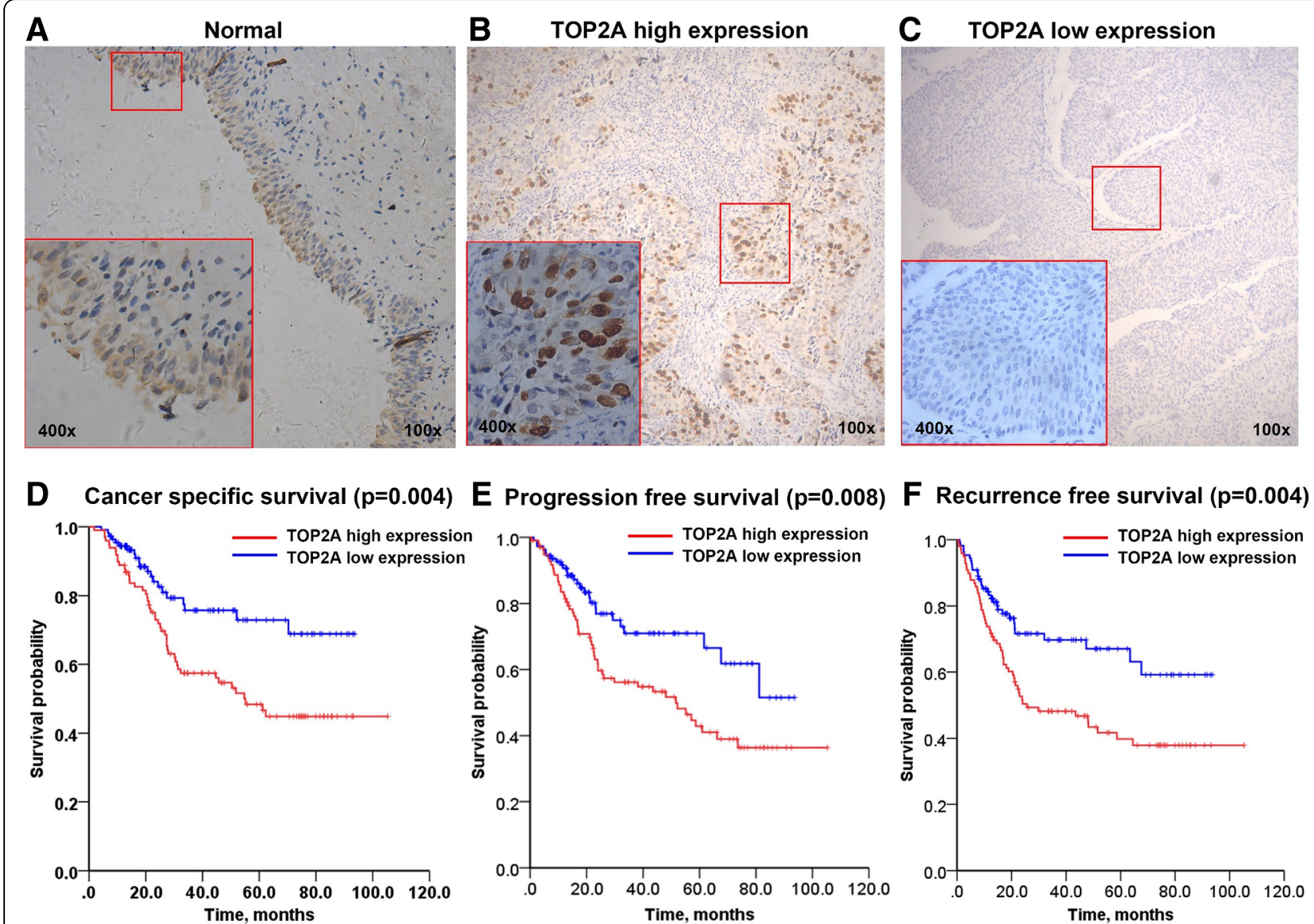

Fig. 2 The relationship between TOP2A protein expression and prognosis of patients with BLCA. a-c Representative images of TOP2A protein expression in normal and bladder cancer tissue. $\mathbf{d}$-f Higher TOP2A protein expression was associated with poorer cancer-specific, progression-free and recurrence-free survival from our single institution cohort $(n=209)$

We also assessed the potential effect of TOP2A knockdown on bladder cancer cell migration and invasion, which were two critical steps accounted for cancer progression and metastasis. Wound healing assay showed that $\mathrm{J} 82$ and 5637 transfected with TOP2A siRNA displayed significantly lower migration capacity compared with control groups (Fig. 4d) - Moreover, the transwell Matrigel invasion assay also indicated that suppression of TOP2A expression strongly suppressed the invasion capacity of J82 and 5637 cells (Fig. 4e). These data suggested that TOP2A was closely associated with migration and invasion capacity of bladder cancer cells.

\section{Apoptosis and doxorubicin resistant induced by TOP2A knockdown}

We performed flow cytometry to compare cell cycle phases and apoptosis in $J 82$ and 5637 cells between TOP2A knockdown and control cells. We found no significant changes in cell cycle phase distribution after TOP2A knockdown in J82 and 5637 cells
(Additional file 3: Figure S3). In terms of apoptosis, knockdown of TOP2A showed a significant increase of early and late apoptosis in both J82 cells and cells after induction of apoptosis by gemcitabine treatment for $24 \mathrm{~h}$ (Fig. 5a). Western-blot assay further indicated the increasing expression of cleaved caspase 3 after TOP2A knockdown (Fig. 5b). These results suggested that TOP2A might play a role of anti-apoptosis in bladder cancer cells. TOP2A is the primary target of doxorubicin, which is an important chemotherapeutic drug for bladder cancer [2]. We thus explored whether TOP2A could serve as a marker of response to doxorubicin in bladder cancer. The sensitivity of TOP2A high expression (J82 and 5637) and low expression (T24 and TCCSUP) cell lines to doxorubicin was compared. According to the CCK-8 assay results (Fig. 5c), TOP2A expression level was not correlated with the sensitivity of bladder cancer cell lines to doxorubicin. Furthermore, we compared the sensitivity of J82 wild type cells (J82/ WT) and J82 cells with TOP2A stably knockdown 
Table 2 Univariable and multivariable COX regression models for predicting cancer specific survival of bladder cancer

\begin{tabular}{|c|c|c|c|c|c|c|}
\hline \multirow[t]{2}{*}{ Variables } & \multicolumn{3}{|c|}{ Univariable } & \multicolumn{3}{|c|}{ Multivariable } \\
\hline & $\mathrm{HR}$ & $95 \% \mathrm{Cl}$ & $P$ value & $\mathrm{HR}$ & $95 \% \mathrm{Cl}$ & $P$ value \\
\hline \multicolumn{7}{|c|}{ Age (<65 yr. as reference) } \\
\hline$\geq 65 \mathrm{yr}$ & 0.79 & $0.48-1.29$ & 0.34 & & & \\
\hline \multicolumn{7}{|c|}{ Gender (female as reference) } \\
\hline Male & 1.07 & $0.63-1.80$ & 0.81 & & & \\
\hline \multicolumn{7}{|c|}{ Pathological stage (T1 as reference) } \\
\hline $\mathrm{T} 2$ & 2.22 & $1.13-4.36$ & 0.02 & 1.67 & $0.83-3.37$ & 0.15 \\
\hline T3 & 4.88 & $2.49-9.56$ & $<0.01$ & 3.32 & $1.62-6.80$ & $<0.01$ \\
\hline T4 & 22.45 & $8.09-62.30$ & $<0.01$ & 16.70 & $5.85-47.69$ & $<0.01$ \\
\hline \multicolumn{7}{|c|}{ Pathological grade (low as reference) } \\
\hline High & 2.33 & $1.19-4.56$ & 0.01 & 1.82 & $0.89-3.72$ & 0.10 \\
\hline \multicolumn{7}{|c|}{ Lymph node (negative or NA as reference) } \\
\hline Positive & 6.14 & $4.17-9.05$ & $<0.01$ & 4.28 & $2.83-6.24$ & $<0.01$ \\
\hline \multicolumn{7}{|c|}{ TOP2A expression (low as reference) } \\
\hline High & 2.09 & $1.25-3.49$ & $<0.01$ & 1.42 & $0.81-2.46$ & 0.21 \\
\hline \multicolumn{7}{|c|}{ Ki67 expression (low as reference) } \\
\hline High & 2.98 & $1.53-5.88$ & $<0.01$ & 2.01 & $0.89-4.60$ & 0.10 \\
\hline
\end{tabular}

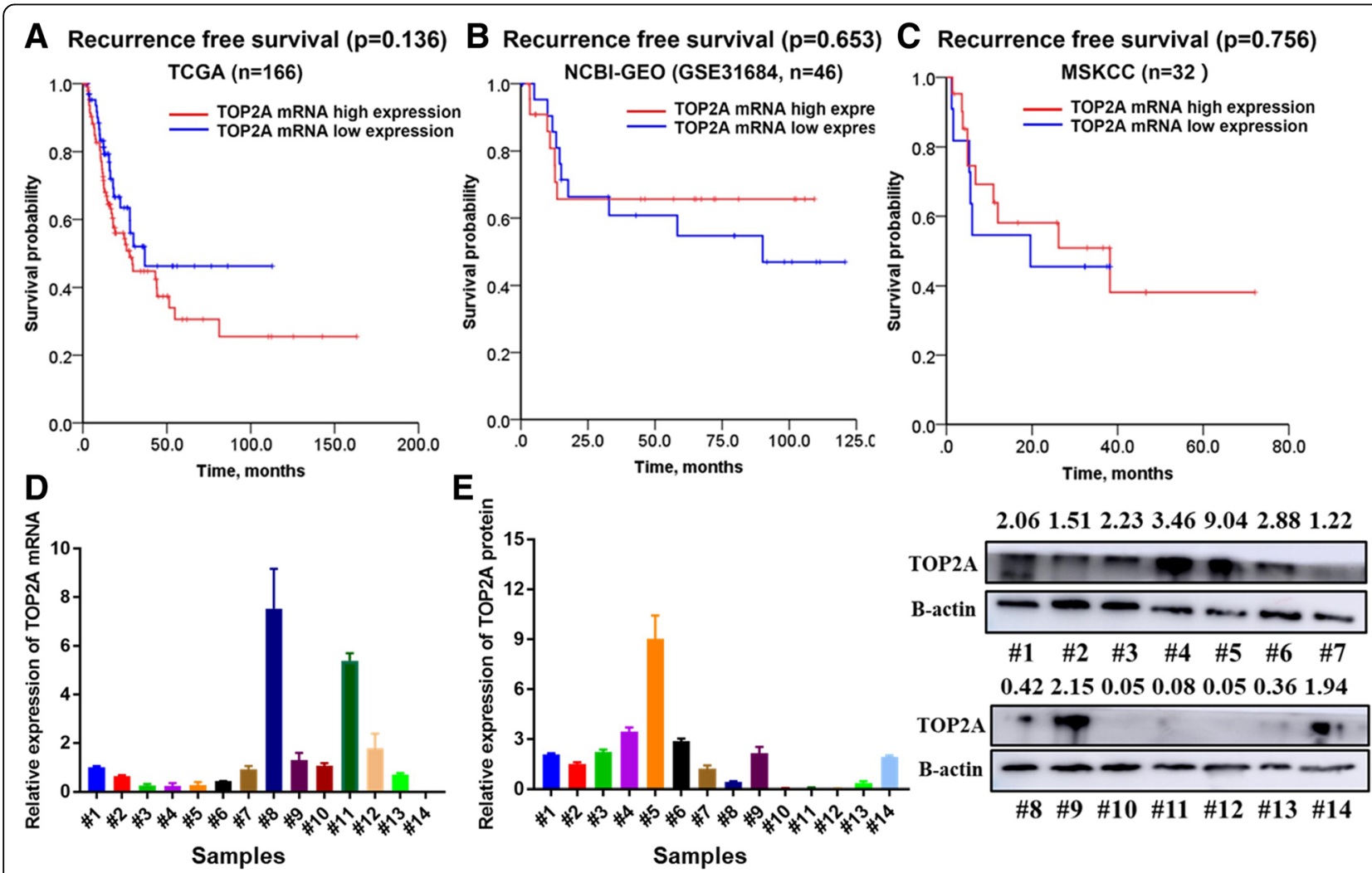

Fig. 3 The prognostic value of TOP2A mRNA and its relationship with TOP2A protein in BLCA. a-c Kaplan-Meier survival curve comparing recurrencefree survival between patients with TOP2A mRNA high and low expression in TCGA, NCBI-GEO (GSE31684) and MSKCC datasets, respectively. Patients with TOP2A mRNA expression above $75 \%$ or below $25 \%$ were included for analysis. $\mathbf{d}$ RT-qPCR assay of TOP2A mRNA expression level in 14 bladder cancer samples. e TOP2A protein level of the 14 bladder cancer samples were investigated by western blotting assay 


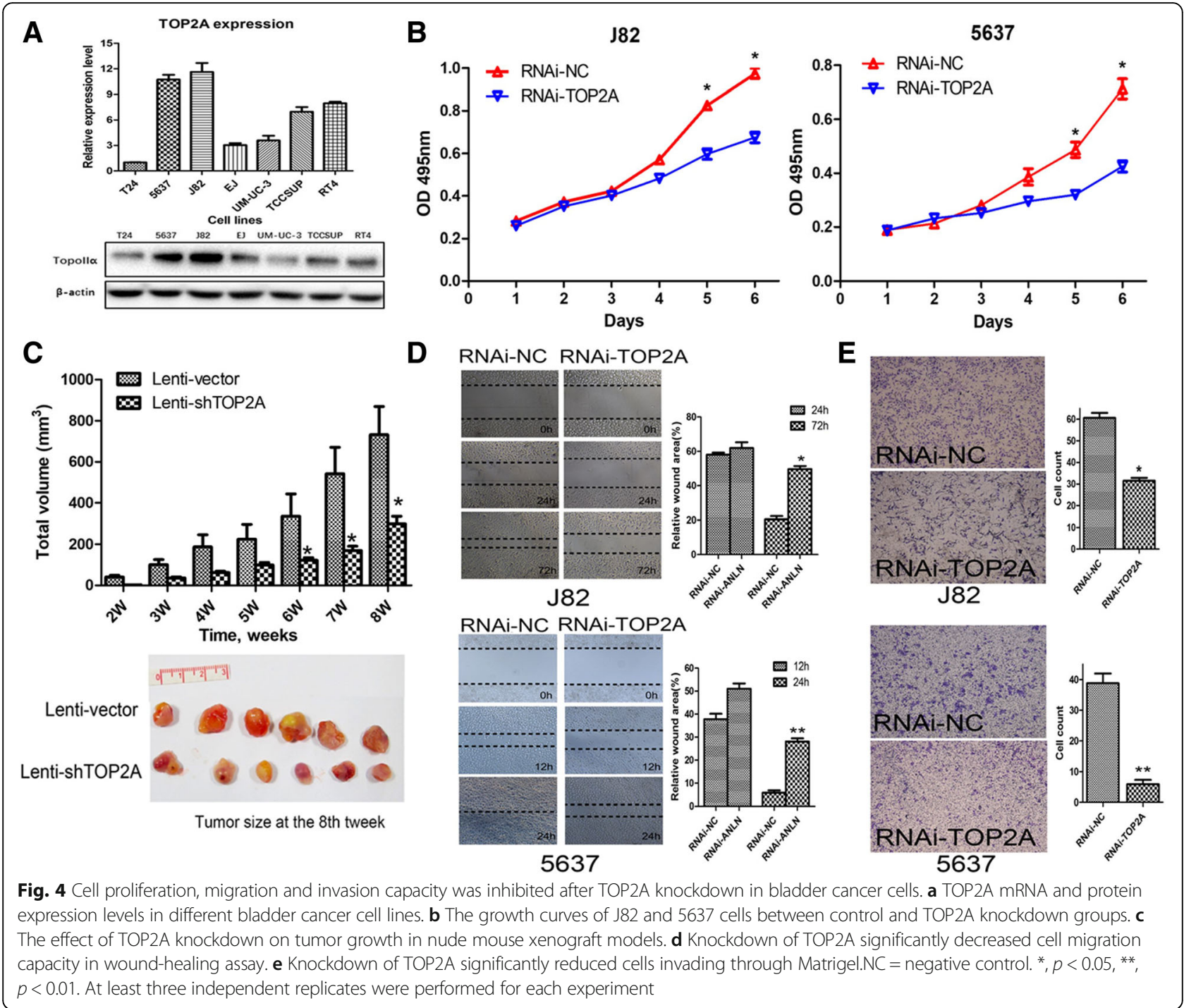

(J82/TOP2A) to doxorubicin. As shown in Fig. 5d, the half maximal inhibitory concentration $\left(\mathrm{IC}_{50}\right)$ values for J82/WT and J82/TOP2A cells were $1.91 \pm$ $0.15 \mu \mathrm{M}$ and $5.74 \pm 0.91 \mu \mathrm{M}$, respectively. J82 cells exhibited relatively resistance to doxorubicin after TOP2A knockdown.

\section{Discussion}

Two types of topoisomerase II enzymes were expressed in mammalian cells, TOP2A and TOP2B, but only TOP2A plays a crucial role for cellular viability $[10,16]$. TOP2A gene, mapped to chromosome 17q12-q21, encodes an enzyme which implicates in almost any processes of DNA metabolism including replication, transcription and chromosome segregation during interphase and mitosis $[17,18]$. TOP2A is thus known to be a marker of cell proliferation in normal tissue, and increased TOP2A expression levels are observed in cancer cells compared with non-malignant cells $[19,20]$. Previous studies also suggested that high TOP2A expression levels could indicate tumor aggressiveness and poor outcome $[3,6,8,21,22]$.

In the present study, our RNA-Seq data of ten pairs of BLCA samples identified TOP2A gene were significantly upregulated in the tumor samples compared with the matched normal epithelial tissue. We further validated that the expression levels of TOP2A mRNA were elevated in tumors by RT-qPCR in a cohort of 40 samples. We found that TOP2A levels were higher in patients with MIBC and highgrade tumors compared with NMIBC and low-grade tumors, respectively. The results were in line with previous studies that TOP2A expression levels reflected the aggressiveness of tumors $[3,6,8,21$, 22]. We further demonstrated that there were significant differences between patients with low- and 


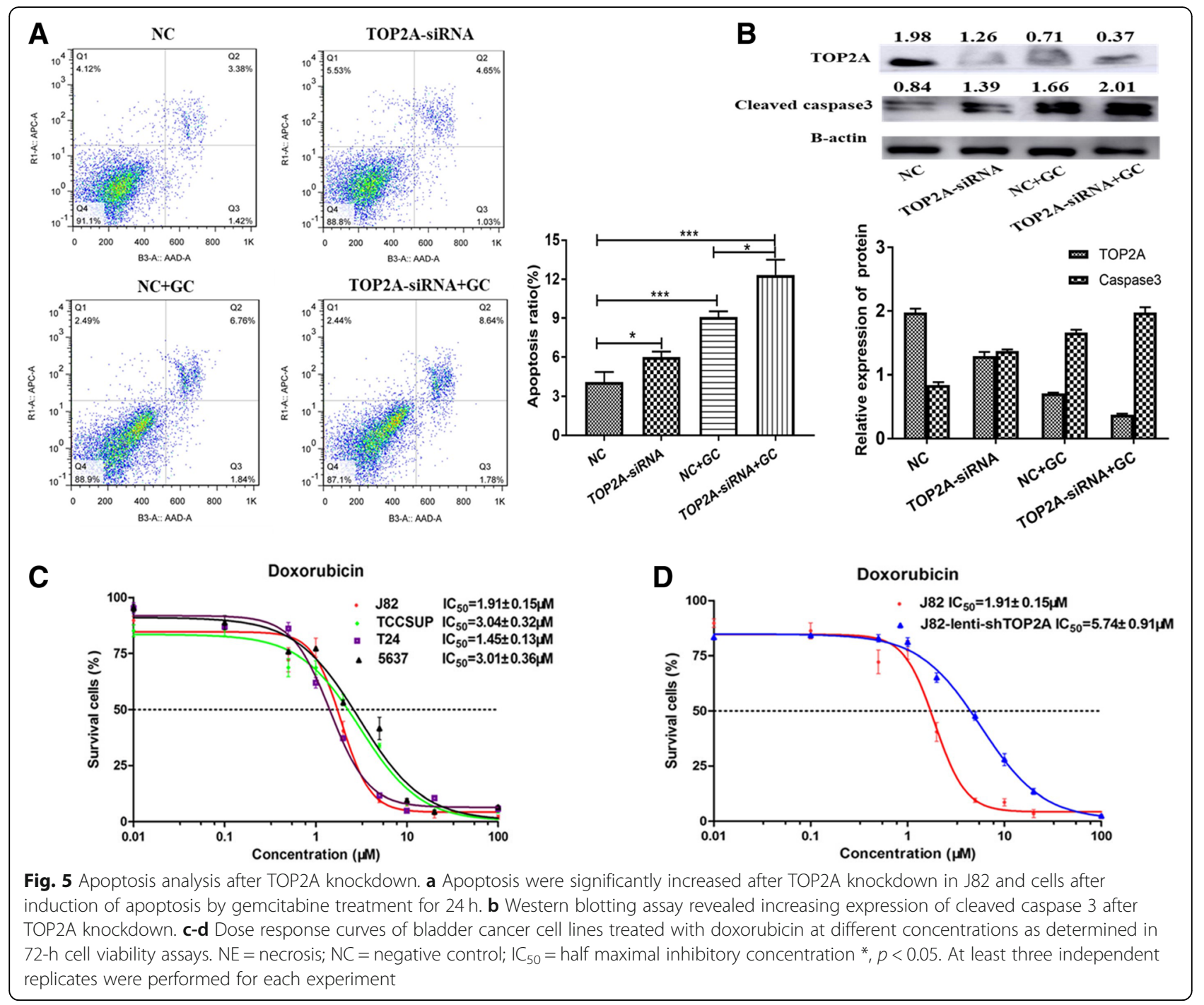

high-expression of TOP2A protein in terms of CSS, PFS and RFS. However, we failed to find TOP2A as an independent risk factor for prognosis in the multivariable COX regression model, and tumor stage and lymph node status served as the most important prognostic factors for BLCA patients. It indicated that TOP2A was an important but not exclusive contributing factor for the progression of BLCA. This result also agreed with several previous studies in BLCA. Simon et al. [23] reported that TOP2A and HER-2 expression was co-amplified in BLCA, and TOP2A amplification were associated with advanced tumor stage and high grade, but they also found no independent prognostic value of TOP2A amplification or protein expression in multivariable COX regression analysis. Koren et al. [24] investigated the expression of TOP2A protein by immunohistochemistry in 57 specimens and found higher TOP2A expression indicated greater probability of
BLCA recurrence and lower overall survival. Subgroup analysis of the prognostic value of TOP2A in MIBC and NMIBC in the present study further revealed that TOP2A had better prognostic value for patients with MIBC rather than NMIBC. This was in contrast to a previous study, Nakopoulou et al. [25] reported that higher TOP2A expression was also indicative of worse prognosis for patients with superficial bladder tumors $(n=51)$. Taken together, TOP2A is a promising biomarker for differentiating patients with different risks of BLCA, and combining TOP2A and conventional prognostic factors, such as tumor stage, may increase the accuracy of predicting prognostic outcomes and provide additional arguments for treatment and surveillance decisions.

Whether the level of TOP2A mRNA could serve as a predictor for the prognosis of BLCA is in controversy. In the present study, public databases analysis showed that patients with higher TOP2A mRNA 
expression did not significantly correlate with the prognosis. We also found no correlation between the level of TOP2A mRNA and protein. It suggests that further studies are warranted to explore the posttranscription mechanisms that contribute to interpret the divergent levels between TOP2A mRNA and protein in BLCA. Meanwhile, Ren et al. [17] revealed high TOP2A expression correlated with worse cancer prognosis, but no relationship between amplification of TOP2A gene and prognosis of cancer in the metaanalysis of 25 studies. In contrast, Kim et al. [3] reported that elevated expression of TOP2A mRNA was indicative of high rate of recurrence and progression in NMIBC $(n=103)$.

Although TOP2A alteration was closely associated with prognosis of BLCA, the oncogenic function of TOP2A in BLCA has not been studied previously. The biological behavior of cancers is generally influenced by two major biological processes: proliferation and invasion [25]. In the present study, we found that knockdown of TOP2A could significantly inhibit the proliferation of bladder cancer cells and non-cancerous urothelial cells, which revealed the essential role of TOP2A in cell proliferation. In addition, knockdown of TOP2A strongly suppressed the migration and invasion capacity of J82 and 5637 cells. Furthermore, flow cytometry analysis suggested that TOP2A played a role in anti-apoptosis in BLCA. These results strengthened the evidence that TOP2A involved in the progression of BLCA. Jain et al. [6] reported that TOP2A was overexpressed in adrenocortical carcinoma and might influence tumor progression, as knockdown of TOP2A in adrenocortical carcinoma cells decreased cell proliferation, and invasion. Gobble et al. [26] found TOP2A was overexpressed in liposarcoma, and knockdown of TOP2A in liposarcoma cell lines reduced proliferation, invasiveness and increased apoptosis. Taken together, TOP2A was proved to play a major role in proliferation and invasion.

Given the essential role of TOP2A in malignant tumors, TOP2A has held the interest of researchers developing targeted anticancer drugs [11]. Doxorubicin and etoposide are two most clinically active anticancer agents targeting TOP2A in different cancers [27-29]. Previous studies suggested that the levels of TOP2A expression could determine the response of chemotherapeutic drugs targeting this enzyme $[10,30,31]$. Tumors with low expression of TOP2A had fewer TOP2A mediated DNA strand breaks induced by targeted drugs and was thus less sensitive than tumors with high level of TOP2A [30]. In the present study, we showed that TOP2A knockdown induced doxorubicin resistance in J82 cells, however, the sensitivity of different bladder cancer cell lines to doxorubicin was not significantly correlated with the expression level of TOP2A. The results implied that the sensitivity of bladder cancer cell lines to doxorubicin might be determined by multiple factors. In addition to TOP2A down-regulation, AbuHammad et al. [32] revealed that metabolizing genes (specifically CYP1A1 and CYP1A2) and other genes were crucial for cell cycle, apoptosis and DNA repair were involved in the development of doxorubicin resistance to breast cancer cells. As a result, combinational biomarkers rather than sole TOP2A expression level warrants further investigation to predict the sensitivity of BLCA to doxorubicin.

\section{Conclusions}

In summary, we identified that the expression of TOP2A was overexpressed in BLCA at both mRNA and protein levels, and TOP2A protein level could serve as a promising prognostic biomarker for BLCA. Furthermore, we found that TOP2A played a major role in BLCA by regulating proliferation, invasion and survival of bladder cancer cells.

\section{Additional files}

\begin{abstract}
Additional file 1: Figure S1. Kaplan-Meier survival curve comparing patients with different expression levels of TOP2A. A Cancer specific survival, progression free survival and recurrence free survival curve between TOP2A high and low expression patients with muscle invasive bladder cancer (MIBC, $n=130$ ). B Cancer specific survival, progression free survival and recurrence free survival curve between TOP2A high and low expression patients with non-muscle invasive bladder cancer (NMIBC) patients ( $n=79)$. (JPG $435 \mathrm{~kb}$ )

Additional file 2: Figure S2. The effect of TOP2A RNA interference on the expression of TOP2A in bladder cancer cells. A Pearson correlation analysis between TOP2A mRNA and protein in bladder cancer samples. B TOP2A was efficiently down-regulated by RNA interference (RNAi) in $J 82$ and 5637 cells. C TOP2B mRNA expression was not influenced by RNA interference (RNAi) against TOP2A in $J 82$ and 5637 cells. D The growth curves of non-cancerous urothelial cells SVHUC showed proliferation rate was inhibited 5 days after TOP2A knockdown. E TOP2A was efficiently down-regulated in $\mathrm{J} 82$ cells after transfection of lentivirus against TOP2A. (JPG 546 kb)

Additional file 3: Figure S3. The effect of TOP2A knockdown on the progression of cell cycle in bladder cancer cells. No significant difference was detected in cell cycle distribution after TOP2A knockdown in $J 82$ and 5637 cells. (JPG 502 kb)

Additional file 4: Table S1. Tumor volumes of subcutaneous xenograft nude mice models in control group and TOP2A knockdown group. This table included the diameter and volume of the tumor sizes in control group and TOP2A knockdown group, which were measured as indicated in the methods section. (XLSX $10 \mathrm{~kb}$ )
\end{abstract}

\section{Abbreviations}

BLCA: Bladder urothelial carcinoma; CSS: Cancer-specific survival; MIBC: Muscle-invasive bladder cancer; NMIBC: Non-muscle invasive bladder cancer; PFS: Progression-free survival; RFS: Recurrence-free survival; RNAseq: RNA-sequencing; RPKM: Reads per gene per kilobase exon per million mapped reads; RT-qPCR: Real time quantitative polymerase chain reaction; TOP2A: Topoisomerase-II alpha; TURBT: Transurethral resection of bladder cancer 


\section{Acknowledgements}

We appreciate the help from Yang Wang and Yongwei Yu, who are from Department of Pathology, Changhai Hospital, China, for analyzing the immunohistochemical staining of samples from patients.

\section{Authors' contributions}

CLX, YHS designed the experiment and supervised the execution of experiments. SXZ, AWL, LHD performed molecular experiments, QX, JY and JSX conducted animal experiments. XWY, ZSZ collected patients' information and performed statistics analysis. SXZ, AWL, LHD, XWY contributed in interpreting the results and drafted the manuscript. SXZ, QX, JY, FL, YPX, JSX revised the manuscript. All authors read and approved the final manuscript.

\section{Funding}

This research was financed by grants from Shanghai Sailing Program (18YF1422700), Shanghai Pujiang Program (18PJD058), Starting Foundation for Young Scientists of Second Military Medical University (2017QN10, 2017QN11), National Natural Science Foundation of China (81772720, 81572509, 81802515, 81801854). The funder did not participate in the design of the study and collection, analysis, interpretation of data or in writing the manuscript.

\section{Availability of data and materials}

The datasets used and analyzed in the current study are available from the corresponding author on reasonable request.

\section{Ethics approval and consent to participate}

Written informed consent was obtained from all patients and all clinical samples obtained were approved by the ethical board of Changhai Hospital. All animal experiments were conducted with the approval of the Scientific Investigation Board of the Second Military Medical University.

\section{Consent for publication}

Not applicable.

\section{Competing interests}

The authors declare that they have no competing interests.

\section{Author details}

'Department of Urology, Changhai Hospital, Second Military Medical University, Shanghai, People's Republic of China. ${ }^{2}$ Department of Geriatrics, Changhai Hospital, Second Military Medical University, Shanghai, People's Republic of China.

Received: 21 August 2018 Accepted: 10 June 2019

Published online: 19 June 2019

\section{References}

1. Chen W, Zheng R, Baade PD, Zhang S, Zeng H, Bray F, Jemal A, Yu XQ, He J. Cancer statistics in China, 2015. CA Cancer J Clin. 2016;66(2):115-32.

2. Alfred Witjes J, Lebret T, Comperat EM, Cowan NC, De Santis M, Bruins HM, Hernandez V, Espinos EL, Dunn J, Rouanne M, et al. Updated 2016 EAU guidelines on muscle-invasive and metastatic bladder Cancer. Eur Urol. 2017;71(3):462-75.

3. Kim EJ, Lee YS, Kim YJ, Kim MJ, Ha YS, Jeong P, Lee OJ, Kim WJ. Clinical implications and prognostic values of topoisomerase-II alpha expression in primary non-muscle-invasive bladder cancer. Urology. 2010;75(6):1516 e1519-3.

4. Network CGAR. Comprehensive molecular characterization of urothelial bladder carcinoma. Nature. 2014;507(7492):315-22.

5. Mitra AP. Molecular substratification of bladder cancer: moving towards individualized patient management. Ther Adv Urol. 2016;8(3):215-33.

6. Jain $M$, Zhang L, He M, Zhang YQ, Shen M, Kebebew E. TOP2A is overexpressed and is a therapeutic target for adrenocortical carcinoma. Endocr Relat Cancer. 2013;20(3):361-70.

7. Erguden HC, Koksal D, Demirag F, Bayiz H, Mutluay N, Berktas B, Berkoglu M. The association of topoisomerase 2alpha expression with prognosis in surgically resected non-small cell lung cancer (NSCLC) patients. J Thorac Dis. 2012;4(4):352-7.

8. Parker AS, Eckel-Passow JE, Serie D, Hilton T, Parasramka M, Joseph RW, Wu $\mathrm{K}$, Cheville JC, Leibovich BC. Higher expression of topoisomerase II alpha is an independent marker of increased risk of cancer-specific death in patients with clear cell renal cell carcinoma. Eur Urol. 2014;66(5):929-35.

9. Depowski PL, Rosenthal SI, Brien TP, Stylos S, Johnson RL, Ross JS. Topoisomerase llalpha expression in breast cancer: correlation with outcome variables. Modern Pathol. 2000;13(5):542-7.

10. Belluti S, Basile V, Benatti P, Ferrari E, Marverti G, Imbriano C. Concurrent inhibition of enzymatic activity and NF-Y-mediated transcription of topoisomerase-llalpha by bis-DemethoxyCurcumin in cancer cells. Cell Death Dis. 2013;4:e756.

11. Nitiss JL. Targeting DNA topoisomerase II in cancer chemotherapy. Nat Rev Cancer. 2009;9(5):338-50

12. Liu A, Zeng $S$, Lu X, Xiong $Q, X u e ~ Y$, Tong $L, X u$ W, Sun Y, Zhang Z, Xu C. Overexpression of $\mathrm{G} 2$ and $\mathrm{S}$ phase-expressed-1 contributes to cell proliferation, migration, and invasion via regulating p53/FoxM1/CCNB1 pathway and predicts poor prognosis in bladder cancer. Int J Biol Macromol. 2019;123:322-34.

13. Zeng S, Yu X, Ma C, Song R, Zhang Z, Zi X, Chen X, Wang Y, Yu Y, Zhao J, et al. Transcriptome sequencing identifies ANLN as a promising prognostic biomarker in bladder urothelial carcinoma. Sci Rep. 2017;7(1):3151.

14. Tarazona S, Garcia-Alcalde F, Dopazo J, Ferrer A, Conesa A. Differential expression in RNA-seq: a matter of depth. Genome Res. 2011;21(12):2213-23.

15. Abbas R, McColl KS, Kresak A, Yang M, Chen Y, Fu P, Wildey G, Dowlati A. PIAS3 expression in squamous cell lung cancer is low and predicts overall survival. Cancer Med. 2015;4(3):325-32.

16. Khelifa T, Casabianca-Pignede MR, Rene B, Jacquemin-Sablon A. Expression of topoisomerases II alpha and beta in Chinese hamster lung cells resistant to topoisomerase II inhibitors. Mol Pharmacol. 1994;46(2):323-8.

17. Ren L, Liu J, Gou K, Xing C. Copy number variation and high expression of DNA topoisomerase II alpha predict worse prognosis of cancer: a metaanalysis. J Cancer. 2018;9(12):2082-92.

18. Warburton PE, Earnshaw WC. Untangling the role of DNA topoisomerase II in mitotic chromosome structure and function. BioEssays : news and reviews in molecular, cellular and developmental biology. 1997;19(2):97-9.

19. Turley H, Comley M, Houlbrook S, Nozaki N, Kikuchi A, Hickson ID, Gatter K, Harris AL. The distribution and expression of the two isoforms of DNA topoisomerase II in normal and neoplastic human tissues. Br J Cancer. 1997; 75(9):1340-6.

20. Lynch BJ, Guinee DG Jr, Holden JA. Human DNA topoisomerase II-alpha: a new marker of cell proliferation in invasive breast cancer. Hum Pathol. 1997; 28(10):1180-8

21. Brase JC, Schmidt M, Fischbach T, Sultmann H, Bojar H, Koelbl H, Hellwig B, Rahnenfuhrer J, Hengstler JG, Gehrmann MC. ERBB2 and TOP2A in breast cancer: a comprehensive analysis of gene amplification, RNA levels, and protein expression and their influence on prognosis and prediction. Clinical cancer research : an official journal of the American Association for Cancer Research. 2010;16(8):2391-401.

22. Kirk JS, Schaarschuch K, Dalimov Z, Lasorsa E, Ku S, Ramakrishnan S, Hu Q, Azabdaftari G, Wang J, Pili R, et al. Top2a identifies and provides epigenetic rationale for novel combination therapeutic strategies for aggressive prostate cancer. Oncotarget. 2015;6(5):3136-46.

23. Simon R, Atefy R, Wagner U, Forster T, Fijan A, Bruderer J, Wilber K, Mihatsch MJ, Gasser T, Sauter G. HER-2 and TOP2A coamplification in urinary bladder cancer. Int J Cancer. 2003;107(5):764-72.

24. Koren R, Kugel V, Dekel Y, Weissman Y, Livne PM, Gal R. Human DNA topoisomerase-llalpha expression as a prognostic factor for transitional cell carcinoma of the urinary bladder. BJU Int. 2003;91(6):489-92.

25. Nakopoulou L, Zervas A, Lazaris AC, Constantinides C, Stravodimos C, Davaris P, Dimopoulos C. Predictive value of topoisomerase II alpha immunostaining in urothelial bladder carcinoma. J Clin Pathol. 2001;54(4):309-13.

26. Gobble RM, Qin LX, Brill ER, Angeles CV, Ugras S, O'Connor RB, Moraco NH, Decarolis PL, Antonescu C, Singer S. Expression profiling of liposarcoma yields a multigene predictor of patient outcome and identifies genes that contribute to liposarcomagenesis. Cancer Res. 2011;71(7):2697-705.

27. Youssef AC. A phase II study of docetaxel followed by vinorelbine and doxorubicin in the neoadjuvant treatment of locally advanced breast cancer. J Clin Oncol. 2009;27(15_suppl):e11542.

28. Vergote I, Finkler NJ, Hall JB, Melnyk O, Edwards RP, Jones M, Meng L, Brown GL, Rankin EM, Burke JJ 2nd, et al. Randomized phase III study of canfosfamide (C, TLK286) plus pegylated liposomal doxorubicin (PLD) versus PLD as second-line therapy in platinum (P) refractory or resistant ovarian cancer (OC). J Clin Oncol. 2009;27(15_suppl):5552. 
29. Flechon A, Pouessel D, Ferlay C, Perol D, Beuzeboc P, Gravis G, Joly F, Oudard S, Deplanque G, Droz J, et al. Results of a phase II study of carboplatin and etoposide in patients with progressive metastatic castration refractory prostate cancer (mCRPC) and neuro-endocrine differentiation. J Clin Oncol. 2009;27(15_suppl):e16073.

30. Davies SM, Robson CN, Davies SL, Hickson ID. Nuclear topoisomerase II levels correlate with the sensitivity of mammalian cells to intercalating agents and epipodophyllotoxins. J Biol Chem. 1988;263(33):17724-9

31. Houlbrook S, Addison CM, Davies SL, Carmichael J, Stratford IJ, Harris AL, Hickson ID. Relationship between expression of topoisomerase II isoforms and intrinsic sensitivity to topoisomerase II inhibitors in breast cancer cell lines. Br J Cancer. 1995;72(6):1454-61.

32. AbuHammad S, Zihlif M. Gene expression alterations in doxorubicin resistant MCF7 breast cancer cell line. Genomics. 2013;101(4):213-20.

\section{Publisher's Note}

Springer Nature remains neutral with regard to jurisdictional claims in published maps and institutional affiliations.

Ready to submit your research? Choose BMC and benefit from:

- fast, convenient online submission

- thorough peer review by experienced researchers in your field

- rapid publication on acceptance

- support for research data, including large and complex data types

- gold Open Access which fosters wider collaboration and increased citations

- maximum visibility for your research: over $100 \mathrm{M}$ website views per year

At BMC, research is always in progress.

Learn more biomedcentral.com/submissions 